\title{
Imagen y medios de comunicación. El caso de los centros hospitalarios en la prensa local.
}

\author{
Image and media. The case of hospitals in the local press.
}

\author{
Mgter. Isabel Ruiz Mora \\ Investigadora y docente \\ Universidad de Málaga (España) \\ Facultad de Ciencias de la Comunicación \\ isabelruiz@uma.es \\ Mgter. Silvia Olmedo Salar \\ Investigadora y docente \\ Universidad de Málaga (España) \\ Facultad de Ciencias de la Comunicación \\ silviaolmedo@uma.es
}

\section{Resumen}

Recibido: 30 de septiembre 2011 Aceptado: 30 de noviembre 2011

En la sociedad actual, las cuestiones de salud cobran cada día una mayor relevancia y los medios de comunicación no son ajenos a ello. En esta línea, partimos de la premisa que las instituciones públicas sanitarias, como son los hospitales, están abordando con una mayor intensidad estrategias comunicacionales para establecer diálogos con sus públicos. Al mismo tiempo los medios de comunicación están otorgando una mayor cobertura al universo sanitario, pues este campo despierta un destacado interés en las audiencias. En concreto, los hospitales, por su contexto de proximidad, se convierten en un elemento de la cotidianidad sobre la que los medios pueden influir de forma decisiva en la construcción de los imaginarios colectivos. Es en este punto, donde esta investigación pretende aportar algunas claves sobre la imagen que proyectan los medios locales sobre los centros hospitalarios y de estar forma, poder constatar los perfiles que caracterizan el protagonismo del centro sanitario y su repercusión mediática, que incidirá de forma significativa en sus públicos. Para poder alcanzar estos objetivos, se utilizará la técnica del análisis de contenido en aquellos periódicos locales de mayor tirada en las capitales de provincia seleccionados para la muestra. Las conclusiones principales nos acercan a la necesidad de que sean las 
propias instituciones las encargadas de despertar el interés hacia las mismas, influyendo así, en la construcción de su imagen.

\section{Summary}

In today's society, health issues are becoming more relevant every day and the media are not alien to it. In this way, we start from the premise that public health institutions such as hospitals are addressing a more intense communication strategies to establish dialogue with their audiences. At the same time, the media is giving more coverage to the medical universe, because this field awakens a strong interest in their public. Specifically, hospitals, for its proximity context, become a part of everyday life on which the media can make a decisive impact on the construction of the collective imagination. At this point, where this paper provides some clues about the image projected on the hospital by local media and through it, try to establish profiles that characterize the role of the health care and media coverage, which will impact significantly in public. To achieve these objectives, we use the technique of content analysis in local newspaper, with the largest circulation, in those selected cities for the sample. The main findings support the conclusion that institutions are responsible for awakening interest to them, influencing the construction of his image.

Palabras Clave: Imagen; prensa local; hospitales públicos; Andalucía; investigación.

Key Words: Image; local press; public hospitals; Andalusia; research.

\section{Introducción}

La presente investigación aborda en un primer estadio un marco conceptual, que guiará la narración teórica del trabajo, para ofrecer posteriormente el desarrollo de la investigación y las conclusiones de la misma. Con este trabajo pretendemos abordar la imagen de los hospitales de Andalucía desde la perspectiva de los periódicos locales más relevantes como formadores y termómetros del pulso de la opinión pública.

El trabajo se enmarca dentro del ámbito de estudio de la proyección mediática de las organizaciones sanitarias de Andalucía, tomando como referencia los principales diarios locales de la región, con el objeto de poder identificar la presencia de los hospitales andaluces en la prensa regional a través de las noticias que se vierten y publican sobre ellos. 


\subsection{Marco conceptual. Imagen versus imagen publicada}

Para abordar esta estudio es preciso que partamos de la distinción de aquellas conceptualizaciones que van a guiar el discurso de la narración como son la imagen y la imagen publicada. Ambas naturalezas de dichos conceptos son diferentes y no deben confundirse ni solaparse. Sin embargo, existe un elemento mediador que incide de forma acuciante en dichos universos conceptuales como son los medios de comunicación y que tiene fuerte repercusiones en el conjunto de las organizaciones.

Losada Díaz (2005) presenta una aproximación a la definición y caracterización de este fenómeno mediador de los medios con el objeto de intentar comprender el impacto que la gestión de comunicación produce en la generación de la imagen de las organizaciones. El autor se refiere a modelos de estudio de la imagen de las organizaciones en los medios de comunicación, o lo que él ha llamado la imagen publicada.

Esta conceptualización (imagen publicada) dista del concepto tradicional de imagen. La imagen es una "representación, estructura o esquema mental que tiene cada público acerca de un objeto (empresa, marca, producto o servicio), conformado por un conjunto de atributos que permiten al sujeto una forma de conocimiento de los ámbitos donde éste vive. No hay que olvidar que la imagen está siempre en el receptor, es un constructo mental propio del mismo" (Benavides, 2001, p.35).

Por lo que podemos concretar que la imagen publicada tiene su fundamento en los discursos plasmados en los medios de comunicación no en las estructuras mentales.

Los medios de comunicación se convierten en exponentes de la escena pública, nutriéndose de las demandas y necesidades de los públicos y de las informaciones generadas por las organizaciones. Actuando como canalizadores del flujo comunicacional y de imagen, conceptualización que a continuación desgranados para una focalización más acertada en su aplicación en el presente trabajo.

Otro punto interesante es el planteo que Losada realiza sobre la formación de la imagen. Al respecto sostiene que la representación de la realidad, que es la imagen, se forma a partir de los estímulos que la persona recibe de determinadas fuentes de información que, de forma general, pueden englobarse en dos categorías diferentes y complementarias: fuentes directas (cuando la información se recibe sin ningún tipo de intermediarios, es decir, a partir de la experiencia personal o del trato directo); o indirectas (cuando la información procede de un intermediario, es decir, se recibe desde un mediador, que puede ser una persona relaciones interpersonales- o los propios medios de comunicación). Los medios de comunicación siempre han representado un vehículo muy atractivo para todo tipo de

Sociedad Latina de Comunicación Social

Facultad de Ciencias de la Información - Universidad de La Laguna

Avenida César Marique, s/n; Campus de Guajara

38071 La Laguna, Tenerife (Islas Canarias - España) 
organizaciones y empresas por su alta capacidad de alcance e influencia, tanto a partir de su dimensión puramente publicitaria (advertising) como de su dimensión informativa (publicity).

En una investigación sobre "La Responsabilidad Social Corporativa de las empresas españolas en los Medios de Comunicación" (Capriotti, 2007) sostiene que diversos autores (Fombrun y Shanley, 1990; Gamson et al., 1992; Wartick, 1992; Fombrun, 1996; Fombrun y Van Riel, 1997 y 2004; Fombrun et al., 2000; Deephouse, 2000; Gardberg y Fombrun, 2002) señalan la presencia en los medios de comunicación cómo visibilidad mediática, constituyéndose como uno de los factores más influyentes en la opinión pública favoreciendo de forma decisiva a la formación de la reputación corporativa que tienen las personas sobre las empresas. Gaines-Ross (1997, p.54) afirma que las compañías con mayor valoración en el Índice de Reputación Corporativa elaborado por la revista Fortune ${ }^{i}$ son aquellas con mayor visibilidad en los medios de comunicación. En este sentido, y en función de lo que venimos planteando, la Media Reputation o Imagen publicada, representa entonces el estado de la misma en los medios de comunicación en un determinado período de tiempo. Al respecto Capriotti (2009) sostiene que los ejes de la discusión se dan en torno a que para realizar una evaluación mediática se debe analizar dos procesos de comunicación: por un lado, el que se genera entre la marca y la relación con los medios en sí, o sea cuál es el tratamiento que recibe de los periodistas; y por otro, el proceso de comunicación que se genera entre el medio masivo y los públicos, es decir, qué lee la gente de lo que dicen los medios sobre una marca o compañía.

Por su parte, José Antonio Alcobeda (2010, p.535) sostiene que la imagen social de las organizaciones entre la opinión pública, dependerá en buena medida de una gestión adecuada y "del correcto tratamiento de la información que generan". Y en concreto, apunta que los organismos públicos, (en este caso los hospitales públicos) "no son entes aislados de esta realidad, por lo que no pueden vivir ajenos a la visibilidad y justificación social de la necesidad de su existencia (Alcoceba, 2010, p.535).

En este marco, recuperamos el concepto de sociedad de pseudoacontecimientos (Losada Díaz, 2005, p.1), que caracteriza a una sociedad "donde las representaciones de la realidad acaban siendo más importantes que la realidad misma". Además, la complejidad informativa y la indiscutible influencia que tienen los medios de comunicación sobre los públicos se ve modificada por la proliferación y desarrollo de las nuevas necesidades informativas, presentando un escenario complejo para los profesionales que se desenvuelven en este campo mediático.

Sociedad Latina de Comunicación Social Facultad de Ciencias de la Información - Universidad de La Laguna

Avenida César Marique, s/n; Campus de Guajara 38071 La Laguna, Tenerife (Islas Canarias - España) 


\subsection{Los medios y la imagen publicada: un modelo de estudio}

En el contexto de la incidencia de los medios como arma poderosa de incidencia en los públicos, se parte de la premisa errónea de los medios como canal - indirecto- de creadores de imagen en los públicos, al producirse una identificación directa y un solapamiento entre imagen e imagen publicada.

El estudio del fenómeno de la imagen publicada puede ser abordado desde perspectivas diferentes en función del momento del proceso productivo de la construcción de textos periodísticos en el que nos situemos. Existen dos fases: el estudio del medio como institución y sus contextos de producción; y el estudio del análisis del mensaje en sí, es decir, el estudio de la forma definitiva adoptada por la noticia, el contenido concreto publicado. Las posibilidades de estudio en esta segunda fase pasan por el análisis de la dimensión puramente formal de la noticia (espacio destinado, ubicación concreta, etc.) y por el análisis de los contenidos transmitidos (temáticas, prioridades, enfoques, presencia de actores y acciones, asociaciones...). Es en esta segunda opción en la que se sitúa el modelo de Losada Díaz y es el que seguiremos en el presente estudio.

Existen también estudios enfocados sobre el impacto o estudio de la recepción del mensaje por su audiencia, es decir, el estudio de los efectos de los medios. Son las consecuencias, evaluadas o supuestas de la acción de las informaciones en la mente de las audiencias.

En este línea, pero incidiendo más directamente en la construcción mental de la realidad abocada por los medios se pronuncia Vázquez Medel (1999), que subraya que "lejos de estar (la realidad) fundamentada en nuestra experiencia directa del mundo, nuestras imágenes de la realidad social provienen de los medios de comunicación" (Vázquez, 1999, p.221). Debido a esta influencia, Vázquez Medel añade:

"Pero no se trata sólo de influir en el contenido de la mente, sino también en nuestras pautas de acción en el mundo. Los medios de comunicación inciden en la construcción no sólo de imágenes inmediatas del mundo (preceptos) o de ideas sobre él (conceptos), sino también en la regulación de nuestra interacción con los seres y las cosas (afectos). No sólo se trata de una incidencia informativa, puramente nocional. Se trata de la construcción de sistemas de valores, de universos axiológicos, y de pautas de acción" (1999, p.223).

En definitiva, los medios operan en la actualidad como los agentes que proporcionan a amplias capas sociales las informaciones y valoraciones que conforman las opiniones sobre las cuales rigen después sus comportamientos y toman sus decisiones. Pero hay que hacer una aclaración, ya que esto no significa, en ningún caso, que podamos hacer el trasvase de

Sociedad Latina de Comunicación Social

Facultad de Ciencias de la Información - Universidad de La Laguna

Avenida César Marique, s/n; Campus de Guajara

38071 La Laguna, Tenerife (Islas Canarias - España) 
los términos de Imagen y de Imagen Publicada de forma gratuita, de manera que puedan considerarse como realidades sinónimas.

En este sentido, el estudio y medición de la Imagen Publicada pasa por entender que es imposible la evaluación completa de la imagen que los medios generan a través de la publicación de los textos si no son escuchados los propios destinatarios de la información, los lectores. A partir de la premisa de que los lectores son los responsables principales de la imagen final que deciden utilizar en el futuro y de que, paralelamente, los medios tienen capacidad para influir en estos lectores en la asignación de los atributos que acaban perfilando esta imagen, esta propuesta quedará en la línea de evaluar objetivamente el material publicado por los medios -al margen de su probable influencia en uno u otro sentidoa partir de una triple perspectiva que da origen a la metodología aplicable posteriormente. Esta triple dimensión se basa en:

- La información que se pone a disposición de los receptores (y, por lo tanto, la que se excluye).

- El modo de presentación de ciertos temas y la relevancia periodística que se les otorga.

- La valoración de las acciones llegadas a cabo por los actores presentes en los textos.

Esta propuesta utiliza los desarrollos propios de la técnica de análisis de contenido, a partir de las aportaciones de autores clásicos como Bardin (1996) o Krippendorff (1990), un material enriquecido en dos vertientes: en primer lugar, en la parte cualitativa del análisis (elección de variables y subvariables) y, en segundo lugar, la codificación estadística de los resultados.

Además, de esta apreciación es necesario apuntar que partimos de la premisa de que en el proceso comunicacional que se genera en los medios de comunicación, están implícitos dos componentes fundamentales que influyen en la alimentación de los imaginarios sociales, como son las objetividad y la parcialidad. La objetividad, entendida como referencia a hechos susceptibles de ser confirmados y contrastados a través de diferentes tipos de fuentes; y por otro la parcialidad, entendida como la toma de posición de cada medio frente a esos mismos hechos. En ese sentido, sostenemos que las producciones periodísticas son parciales (reflejan tomas de partido a favor o en contra de determinados intereses de clase o grupos) y tienen como objetivo final la creación de sentidos comunes hegemónicos.

Ello también nos lleva a discernir en las aportaciones vinculadas a las unidades informativas como son la textual, limitada a la unidad de actuación lingüística, y el contexto -unidad

Sociedad Latina de Comunicación Social Facultad de Ciencias de la Información - Universidad de La Laguna

Avenida César Marique, s/n; Campus de Guajara 38071 La Laguna, Tenerife (Islas Canarias - España) 
extratextual de interpretación sociocultural que forma parte de los niveles cognitivos de quien recibe la información- como subraya Losada Díaz (2002), siendo esta última dimensión la que afecta y confiere a los textos una incidencia diferente en cada receptor y de forma extensiva se prolonga al grupo de individuos que configuran un mismo público atendido por la organización. En este caso que planteamos, esos públicos no reciben la información de forma directa, sino mediatiza por el medio de comunicación, siendo un portavoz que escapa al control de la propia organización.

Estos valores inciden de forma directa en otro ingrediente que es la credibilidad, el gran basamento de los medios de comunicación y que, según mantiene Losada Díaz (2002), contribuye al fortalecimiento de la imagen de las organizaciones en el imaginario colectivo a través de aquellas referencias que se plasman en la prensa.

"La credibilidad es un punto de arranque muy valioso para la consecución de apreciaciones, opiniones y percepciones sobre una organización, al ser los medios de comunicación un soporte que no supone para el receptor externo -y hasta para el interno- ninguna relación con los intereses de la organización, ninguna voluntad propagandística que trate de ocultar la verdadera realidad" (Losada Díaz, 2002, pp.104-105).

Por lo que se incide en que los medios van a ser una plataforma de consolidación de las identidades de las organizaciones, en este caso las sanitarias, de cara al conjunto de los públicos que se nutren de su servicio y que se ven afectados por sus representaciones en el universo mediático y en su repercusión colectiva.

De hecho, en el caso de las instituciones públicas se constata ciertas cambios como indica Alcobedas (2010, p.535) al señalar que en "los últimos tiempos las instituciones públicas han ido tomando conciencia de la importancia del tratamiento y la gestión de la información interna, y de su difusión externa hacia las audiencias a través de los medios de comunicación".

\subsection{La comunicación en las organizaciones sanitarias}

Partimos de la premisa que los hospitales son organizaciones y si concebimos a las organizaciones como sistemas, implica una interdependencia de sus elementos y la comunicación se posiciona como el factor que integra sus componentes y

"es esencial para su desarrollo pues a través de ella, se definen, establecen, comparten y retroalimentan la misión, visión, valores, programas, planes y proyectos; 
estrategias, objetivos, políticas, normas, reglas, actividades y resultados, entre otros" (Arras Vota et ad, 2008, p.418)

Por tanto, la gestión de la comunicación en las organizaciones sanitarias debe contemplarse de vital importancia para el correcto funcionamiento de dicha institución. De igual modo que se otorga un papel principal a la profesionalidad de los facultativos, a la innovación de las técnicas empleadas o a la calidad del servicio prestado, una buena cohesión comunicativa repercutirá positivamente no sólo en el ámbito interno sino que reforzará la imagen de la organización en el exterior. Será la actitud de los públicos claves (stakeholders) para la organización la que determine el buen hacer de la política comunicativa de la institución, entre otros grupos de interés, nos referimos concretamente a los medios de comunicación y a los ciudadanos (usuarios y/o pacientes), éstos últimos mediados en cierta forma por la información que reciben a través de determinados medios, como es la prensa.

La presencia cada día más usual y constante de información especializada en salud o información sanitaria está motivada, entre otros motivos, por (Becerra, 2006):

- La demanda de la sociedad general sobre informaciones relacionadas con dichas instituciones (avances médicos, negligencias, pacientes, ...).

- La proliferación de gabinetes y departamentos de comunicación en estas instituciones, principalmente a partir de los años 90 , con el fin no sólo de gestionar la información que se publicaba sobre la organización, sino también su imagen.

- La importancia que tiene este tipo de información en la agenda de los medios de comunicación, sobrepasando en muchas ocasiones el ámbito local y ocupando en muchos casos secciones fijas o suplementos dentro de los propios medios.

Aunque como hemos mencionado existen otros muchos motivos, estos tres hacen que el binomio hospital local - medio local adquiera gran protagonismo para este estudio, por la repercusión que las noticias sobre esta institución puedan tener sobre el público externo más inmediato de esta organizaciones, los usuarios y/o pacientes.

En el ámbito público español el origen e importancia de las relaciones públicas (y en consecuencia, de la comunicación organizacional) "se sitúa a partir de 1956, año en que el que se producen importantes cambios en el país, que perseguían la incorporación de técnicas modernas de racionalización del trabajo y resolver los problemas de comunicación social" (Almansa, 2010, p.3). Poco a poco la administración pública asimiló la importancia de la gestión de las relaciones con sus públicos y transmitiendo dicho interés a todos sus ámbitos de actuación, incluido el sanitario. De ahí la reciente proliferación de gabinetes de comunicación en las instituciones públicas.

Sociedad Latina de Comunicación Social Facultad de Ciencias de la Información - Universidad de La Laguna

Avenida César Marique, s/n; Campus de Guajara

38071 La Laguna, Tenerife (Islas Canarias - España) 
Podemos hablar de dos momentos históricos actuales que han caracterizado la gestión de la comunicación en las organizaciones sanitarias (Becerra, 2006).

- Los años 90 del pasado siglo, cuando comienza a aplicarse un enfoque empresarial en el sector sanitario español. Caracterizados por tres cambios producidos en el sector: comienza a visualizarse al usuario/paciente como cliente; las instituciones sanitarias comienzan a preocuparse por el medio en el que están integradas, por sus públicos y opiniones; implicación de los empleados en los objetivos y estrategias de la organización. Estos tres cambios motivan la aparición de gabinetes de comunicación, líneas estratégicas de comunicación o gestión de conflictos. En esta misma década aparecen en los principales diarios españoles las secciones especializadas en salud con periodicidad semanal (Costa Sánchez, 2008).

- A partir del año 2000 comienza a producirse un auge de la profesionalización de la comunicación en el sector que nos ocupa, creando unidades o departamentos de comunicación para la implantación de rutinas de trabajo. Como consecuencia se produce un mayor interés por conocer a los públicos; aumenta el interés por conocer la experiencia del usuario/paciente en la institución; existe una verdadera necesidad de colaboración con los medios de comunicación. Estos cambios de actitud están orientados a mejorar el servicio que se presta y mejorar o mantener la imagen de la organización entre sus públicos.

Es de destacar la importante labor que los gabinetes o departamentos de comunicación realizan como mediadores de la organización sanitaria con sus públicos, como González (2004), menciona en su artículo "Salud, información periodística en alza" la gestión de la comunicación y por ende, los gabinetes de comunicación, suponen un

"valor añadido, se obtiene una presencia regular en los medios de comunicación, la confianza cada vez mayor de los ciudadanos y la transmisión de informaciones anteriormente no difundidas a los usuarios potenciales de la sanidad. En este sentido, hay que valorar positivamente la germinación de gabinetes de comunicación en las distintas instituciones y servicios sanitarios (...), pues mediante su labor dan a conocer a la población información de interés y, al mismo tiempo, facilitan el trabajo a los periodistas de los medios de comunicación social en un entorno muy cerrado" (2004, p.308).

Sobre todo es de destacar la labor que realizan los profesionales de la comunicación como intermediarios entre la organización y los medios de comunicación locales, trabajando en pro de la imagen de la organización, tanto en situaciones normales como en las más críticas por 
un lado y por otro, actuando de manera cercana para los periodistas, a los que se les facilita su trabajo suministrándoles información y datos, convirtiéndose en fuentes de información fiables y contrastables.

En esta relación con los medios de comunicación, se deben tener en cuenta ciertas premisas que pueden afectar negativamente a la organización, institución, empresa o administración pública y que afectan directamente a las rutinas de los departamentos o gabinetes de comunicación. El gabinete de comunicación se postula como un emisor de información fiable pero no podemos caer en la tentación de convertirnos en protagonistas de los diarios en la prensa, como Álvarez y Caballero (1997) mencionan "la omnipresencia no suele ser sinónimo de calidad"1 , lo mismo podemos apuntar en cuanto a la adecuación del contenido informativo al medio, la relevancia del mismo y el grado de interés, entendiendo por tal actualidad, cercanía, proximidad al público (Almansa, 2010). Desde la organización se debe dar especial importancia a la veracidad, transparencia y profesionalidad en la relación con los medios (Ramírez, 1995) ${ }^{2}$.

Pero sí es preciso tener en cuenta, como afirma Idoia Camacho (2001), diferentes parámetros en la estrategia global de comunicación de la organización. Según Camacho (2001), se debe canalizar las informaciones que nacen de las distintas áreas de la organización, e insiste en que dicha comunicación global "debe integrar y armonizar la comunicación interna y la externa de la institución. Se trata de definir una imagen coherente que encaje con la finalidad de la organización y la potencie".

\subsection{Objetivos}

El trabajo persigue dar respuesta a tres objetivos principales, que van a guiar el desarrollo de la investigación:

- Determinar cuáles son las principales informaciones que aparecen en los diarios de Andalucía sobre estas instituciones

- Analizar el estado de la comunicación de dichas organizaciones con el periódico local de referencia

- Identificar la proyección de la imagen de los hospitales de la muestra a través de la prensa

\footnotetext{
${ }^{1}$ Citado por Almansa, A. "Relaciones Públicas y Medios de Comunicación. Estudio del caso Español", en Razón y Palabra, 70, 2010.

${ }^{2}$ Citado por Almansa, A. "Relaciones Públicas y Medios de Comunicación. Estudio del caso Español”, en Razón y Palabra, 70, 2010.
}

Sociedad Latina de Comunicación Social Facultad de Ciencias de la Información - Universidad de La Laguna

Avenida César Marique, s/n; Campus de Guajara

38071 La Laguna, Tenerife (Islas Canarias - España) 
Se vehiculan estos objetivos partiendo de la base de la interrelación que se producen entre los medios de comunicación, en este caso, la prensa local, los centros hospitalarios y la ciudadanía, que es la receptora última del fluido comunicativo.

\section{Método}

La metodología llevada a cabo para realizar este estudio se ha basado en la técnica cuantitativa del análisis de contenido. Con esta técnica podremos estudiar y comprender la presencia de las organizaciones sanitarias seleccionadas en los medios elegidos.

Esta técnica ha sido definida como "la técnica que supone aplicar sistemáticamente unas reglas fijadas previamente que sirvan para medir la frecuencia con que aparecen unos elementos de interés en el conjunto de una masa de información que hemos seleccionado para estudiar alguno de los aspectos que nos parecen útiles conforme a los propósitos de nuestra investigación" (Berganza y Ruiz, 2005, p.213). Otros autores, como Krippendorff señalan que análisis de contenido "debe ser predictivo de algo en principio observable, que facilite la toma de decisiones o que contribuya a conceptualizar la porción de la realidad que dio origen al texto analizado. Cualquier análisis de contenido debe realizarse en relación con el contexto de los datos" (1990, p.32).

Esta herramienta nos permitirá acercarnos pertinentemente a nuestro objeto de estudio, para poder obtener unas conclusiones caracterizadas por la fiabilidad y la veracidad de los resultados derivados del análisis.

\subsection{Muestra}

La muestra de nuestro estudio está compuesta por los hospitales públicos andaluces que atienden a un mayor sector poblacional y la presencia de éstos mismos en los principales diarios locales. Para la elección de los Centros Hospitalarios de mayores dimensiones, perteneciente a la Administración Pública, que atienden a un mayor número de habitantes, se tuvo en cuenta las provincias de Andalucía ordenadas según el número de habitantes censados en cada una de ellas ${ }^{3}$.

\footnotetext{
${ }^{3}$ Poblaciones referidas al 1 de enero de 2009 por provincias y sexo (Unidades: Personas). Real Decreto 1918/2009, de 11 de diciembre.
}

Sociedad Latina de Comunicación Social Facultad de Ciencias de la Información - Universidad de La Laguna Avenida César Marique, s/n; Campus de Guajara 38071 La Laguna, Tenerife (Islas Canarias - España) 


\begin{tabular}{|l|l|}
\hline Provincia & Total \\
\hline Sevilla & 1.900 .224 \\
\hline Málaga & 1.593 .068 \\
\hline Cádiz & 1.230 .594 \\
\hline Granada & 907.428 \\
\hline Córdoba & 803.998 \\
\hline Almería & 684.426 \\
\hline Jaén & 669.782 \\
\hline Huelva & 513.403 \\
\hline
\end{tabular}

Así, elegimos las tres primeras provincias andaluzas y los hospitales públicos de dichas ciudades por ser éstas las más pobladas, superando la barrera del millón de habitantes. Por tanto, los centros hospitalarios analizados se corresponden con las ciudades de Sevilla, Málaga y Cádiz. En el caso de Sevilla, se eligió el Hospital Universitario Virgen del Rocío; en Málaga, el Hospital Regional Carlos Haya y en Cádiz, el Hospital Puerta del Mar.

Conjuntamente se eligió el diario local con mayor tirada, difusión y audiencia de cada provincia, por lo que la relación de diarios es la siguiente:

\begin{tabular}{|l|l|}
\hline Provincia & Diario Local \\
\hline Sevilla & Diario de Sevilla \\
\hline Málaga & Diario Sur \\
\hline Cádiz & Diario de Cádiz \\
\hline
\end{tabular}

\subsection{Modelo de Estudio}

De acuerdo con la técnica de investigación que hemos empleado en este estudio se ha desarrollado un cuadro de análisis en el que registraremos las noticias aparecidas sobre los hospitales seleccionados en los diarios elegidos. El diseño del cuadro de análisis se ha elaborado en función de tres objetivos principales: 
- Conocer los temas con los que usualmente aparecen relacionados los hospitales en los medios locales.

- Determinar la calidad de las noticias para poder realizar una valoración de los artículos publicados.

- Definir el conjunto de valores asociados en la proyección mediática de los centros sanitarios.

Dicha herramienta se adecua a nuestras principales variables de estudio:

- Notoriedad

- Tratamiento del medio

- Fuentes informativas

- Agenda de temas vinculados

- Públicos afectados

- Ámbitos de interés

- Calidad de la información

- Valoración y percepción del analista

Estas variables se agrupan en torno a dos ejes principales:

a) Datos técnicos, que engloba información relacionada con la fecha, ubicación, extensión, elementos gráficos, fuentes o sección donde aparecen las noticias, reportajes y otras informaciones.

b) Datos valorativos, que recogen las informaciones referentes a formato, tema que aborda la unidad de análisis, públicos afectados por la información, continuidad de las informaciones, ámbito de interés, calidad de la información y valoración y percepción personal del analista.

Para la selección de las noticias, se ha determinado como criterio la aparición del nombre del complejo sanitario estudiado en el titular o en el subtítulo del periódico al considerar que este hecho nos va a permitir obtener datos más relevantes de la presencia y relevancia pública del Hospital. Además, teniendo en cuenta que los titulares son casi las únicas secuencias leídas por gran parte de los lectores de diarios, como sostienen numerosas 
investigaciones como las de García \& Stark (1991) ${ }^{4}$, nos proporcionarán unos resultados más ricos en contenidos y conclusiones más sólidas.

El análisis se llevó a cabo durante el primer trimestre del año 2010, exceptuando el caso del Hospital Puerta del Mar, cuyo análisis se amplió a dos trimestres (último del 2009 y primero del 2010) por no aparecer un número significativo de noticias para el estudio. Este período ha pretendido no ser interrumpido por la época estival que al aumentar el número de población puede influir en la incidencia mediática de las apariciones de los centros hospitalarios.

Siempre tomando como eje de análisis las exposiciones con mención explícita de los distintos hospitales, los valores están expresados en "cantidad de noticias".

\section{Resultados}

Los resultados son presentados en función de las variables que han determinado el análisis e individualizados para cada hospital estudiado. Con esta estructura podremos realizar un análisis de cada caso y una posterior comparación para poder alcanzar unas conclusiones comunes del estudio.

\section{Diario de Sevilla y Hospital Universitario Virgen del Rocío}

A continuación ofrecemos los resultados obtenidos del análisis llevado a cabo en las noticias publicadas sobre el Hospital Universitario Virgen del Rocío en el Diario de Sevilla.

En cuanto a las secciones en las que cobra mayor protagonismo las noticias relacionadas con el hospital Virgen del Rocío, destacamos Portada (12\%), Sevilla (52\%), Sociedad (4\%), Vivir en Sevilla (20\%) y Opinión (12\%).

Resaltamos que el $64 \%$ de las noticias aparecen en página impar, frente al $36 \%$ que lo hace en una página par.

El 32\% de las noticias aparecen sin un apoyo gráfico que realce la atención de la misma en la sección del periódico en la que se encuentra ubicada y un 36\% aparece sin subtítulo.

Los formatos predominantes son noticias con un $80 \%$ y a una mayor distancia el artículo de opinión con un $12 \%$ y reportaje y entrevista con un $4 \%$ respectivamente.

${ }^{4}$ Citado por Sánchez,1990. "Títulos y titulares. Sobre las funciones de la titulación periodística”, Comunicación y Sociedad, No 1 y 2, Volumen III, pp. 173-183.

Sociedad Latina de Comunicación Social Facultad de Ciencias de la Información - Universidad de La Laguna

Avenida César Marique, s/n; Campus de Guajara

38071 La Laguna, Tenerife (Islas Canarias - España) 
Entre las fuentes de las que proceden las noticias publicadas, destacamos que en el 92\% de los casos se presenta el propio periódico como agente creador de información, seguido de agencia y cartas al director con un $4 \%$ en ambos casos.

Los temas sobre los que versan las noticias publicadas se centran en un 68\% sobre innovación y avances científicos, el restante 32\% versa sobre temas enmarcados en el apartado "otros", entre ellos destacamos noticias relacionadas con:

- Infraestructuras

- Voluntariado

- Publicaciones

- Atención a pacientes

- Donación de sangre de directivos

- Gestión

- Muestras artísticas de empleados

Los públicos afectados por las noticias, protagonistas o no de las mismas, son la propia organización (88\%), los empleados (76\%), los pacientes (68\%), usuarios (20\%), la Administración Pública (4\%) y otros colectivos (8\%).

Destacamos el hecho de que el grupo organización + empleados + pacientes/usuarios aparecen como protagonistas de la misma noticia en el $48 \%$ de las noticias y el grupo, organización + empleados en el 16\% de las noticias analizadas.

Los ámbitos de interés que abarcan las noticias estudiadas son en su mayoría internacional (44\%), seguido de local (28\%), nacional (20\%) y regional (8\%).

La calidad de las noticias publicadas es, en un $84 \%$ divulgativa y en un $16 \%$ versan sobre un acontecimiento puntual que tiene lugar en el hospital o que está relacionado con éste.

El cuanto a la valoración de las noticias analizadas, en un $84 \%$ es positiva, en un $12 \%$ neutra y en un $4 \%$ negativa. Como vemos la percepción general tiene unos resultados bastante similares, positiva un $88 \%$, neutra un $8 \%$ y negativa con un $4 \%$.

\section{Diario Sur y Hospital Carlos Haya de Málaga}

A continuación ofrecemos los resultados obtenidos del análisis llevado a cabo en las noticias publicadas sobre el Hospital Carlos Haya en el Diario Sur. 
En el período analizado, el hospital presenta una media de 1 a 2 repercusiones por semana. Respecto del protagonismo informativo, casi el 50\% de las exposiciones analizadas tenían al hospital con objeto principal de la información, ocupando página completa. En estos casos, como veremos más adelante complementada con apoyo visual y subtítulo.

Un $20 \%$ de las exposiciones aparecen en la página 9 del diario, y un 14\% en la página 8. El resto de las páginas con más de una exposición corresponden a la 11, 13, 14 y 16.

El 61\% son de formato "noticia, y un 13\% son de opinión. El resto corresponde a reportajes o entrevistas.

El apoyo visual y los subtítulos están presentes en casi el 50\% de las exposiciones.

En el total de noticias analizadas no es posible determinar otra fuente que no sea el propio medio, por otra parte, no se detallan agencias de noticias. Es de destacar, que muchas de las notas aparecen firmadas por el periodista Ángel Escalera; sin embargo, por el contenido, en su mayoría divulgativo y positivo, es posible deducir que las mismas provienen de la propia organización.

Según la nube de tags que arroja el análisis, la temática con la que el hospital sale expuesto en Diario Sur, se vincula a temas de innovación de manera principal. También aparecen temas vinculados a cuestiones sindicales y a la formación de los empleados o el personal del nosocomio.

En el 65\% de las exposiciones, la organización es el principal público objetivo. El resto se distribuye equitativamente entre usuarios, otros colectivos (vecinos de la zona), empleados y la Administración Pública. El 83\% de las repercusiones analizadas son del ámbito regional, por ser el Hospital Carlos Haya, un centro de referencia a nivel regional.

En cuanto a la calidad de la información y la adjetivación periodística con la que las exposiciones son tratadas, nos encontramos con una amplia mayoría (70\%) de informaciones divulgativas que encuentran una correlación con las valoraciones de las mismas, ya que en un $70 \%$ son positivas. En este sentido, cómo ya destacáramos anteriormente, se ven reflejadas muchas exposiciones "aparentemente gestionadas" desde el mismo centro. Las exposiciones del tipo conflicto, están principalmente asociadas a denuncias sindicales o contra la Administración Pública, y conllevan una adjetivación negativa para con el Hospital Carlos Haya, en su tratamiento y valoración periodística.

\section{Diario de Cádiz y Hospital Puerta del Mar}

En un análisis general de los resultados arrojados por el estudio, se observa que la sección Cádiz, es la que recoge más información relacionada con el complejo hospitalario (56\%), 
seguida de Provincias (24\%). mientras que la ocupación de apertura a lo largo de casi un año se produce en sólo dos ocasiones (8\%). En la sección de Opinión, la aparición es casi testimonial (8\%) mientras que en Sanidad queda reducida a una única aparición (4\%).

Un $72 \%$ de las informaciones analizadas tienen apoyo gráfico, otorgándole un mayor protagonismo al tema relacionado con el complejo sanitario, de las cuales un $36 \%$ muestran directamente aspectos particulares del hospital como el edificio, salas de espera y otras partes del mismo. Un $8 \%$ corresponden a empleados del hospital y un $28 \%$ a otros espacios y personajes que, de una u otra forma, quedan vinculados a la información facilitada.

Cabe mencionar, que la dimensión de la mayor parte de las noticias supera la media página, espacio que se ha visto favorecido por el criterio de selección de noticias -aparición del nombre del hospital en el título y subtítulo- y del recurso gráfico.

La noticia es el género más utilizado (80\%) en las informaciones facilitadas, hecho que se corresponde con la propia naturaleza del medio estudiado, un periódico diario, en consonancia a los principios de actualidad y al criterio de interés social. No obstante, da cabida a otros géneros como es el reportaje (8\%), opinión (8\%) y la entrevista (4\%).

Sin duda, es el propio periódico a través de sus periodistas los que elaboran y gestionan la mayoría de las informaciones proporcionadas (56\%). Sin embargo, existe otro elenco de fuentes informativas que son las que proporcionan la información: el propio hospital (20\%), la Junta de Andalucía mediante el Servicio Andaluz de Salud (SAS) (16\%), partidos políticossindicatos (8\%) y particulares- cartas al director (4\%).

En cuanto a la temática, las informaciones producidas por el hospital tienen un amplio abanico de asuntos. Destaca el hecho de las informaciones referentes a negligencias médicas y procesos judiciales, que alcanzan el 16\%. Esta cifra se produce a pesar de la extensa gama de temas existente, logrando la cota más alta junto al apartado de "otros", que incluye noticias de diversa índole.

Por otra parte, la innovación, la formación sanitaria, los sucesos y los casos de gripe/contagio alcanzan cada una de las áreas citadas un 12\%, mientras que las cuestiones laborales así como asuntos relacionados con la política o sindicatos representan un $8 \%$ respectivamente.

La propia organización y los pacientes son los principales públicos afectados en las noticias publicadas con un $88 \%$ y $80 \%$, respectivamente, frente al resto de públicos pertinentes como son los usuarios-no hospitalizados (16\%), otros colectivos (20\%), empleados (8\%) y administraciones públicas (8\%). 
El ámbito local-provincial se erige como el referente territorial de las informaciones (80\%), aunque también tiene lugar noticias que logran la proyección regional (4\%) y la nacional $16 \%)$, éste último, logrado por temas vinculados a personajes de relevancia en el panorama político nacional como Julio Anguita (ex coordinador nacional de Izquierda Unida) o a cuestiones de la Gripe A, que afectó a todo el país.

La calidad divulgativa de las informaciones (40\%) es la tónica más generalizada de las noticias analizadas, seguida de cuestiones en las que el conflicto es el eje de la información (28\%), equiparándose los asuntos institucionales y sensacionalistas con un $12 \%$ ambas, mientras que las interpretativas representan el $8 \%$.

Las valoraciones generales de los titulares y del conjunto de las informaciones responden al análisis realizado. En este sentido, podemos señalar que en el apartado de los titulares, el hospital únicamente obtiene un $40 \%$ de valoración positiva mientras que la negativa alcanza un $32 \%$ y la neutra el $28 \%$.

En un sentido más amplio y general de las informaciones recogidas, la percepción sigue criterios semejantes que prácticamente coinciden con la valoración de los titulares. En este orden de cosas, se obtiene un reflejo positivo en el $52 \%$ de los casos, negativo en el $28 \%$ y neutro en el $20 \%$.

\section{Conclusiones y discusión}

El estudio realizado nos permite plantear una serie de conclusiones que se desprenden de los datos obtenidos y que nos aproxima a la proyección actual en los periódicos locales de dichas instituciones públicas, mediación que contribuye a construir el imagino colectivo de la ciudadanía respecto a dichas organizaciones.

- Las secciones en las que aparece un mayor número de noticias relacionadas con el hospital son las dedicadas a la información local. Con esta situación nos hacemos eco de la importancia que el periódico otorga a las informaciones relacionadas con el hospital, puesto que las ubica en las secciones que recogen las noticias que tiene una mayor importancia para el ciudadano, ya que hablan de la ciudad donde residen, lo ocurre en ella y que por tanto, les afecta de manera directa.

- Esta importancia queda igualmente reflejada en las páginas en las que aparecen ubicadas dichas noticias, en su mayoría son las páginas impares y son éstas, las que captan una mayor atención de los lectores, otorgándoles por tanto una mayor relevancia. 
Esta situación se repite con la presencia de elementos gráficos (fotografías e infografías) que resaltan aún más esa llamada de atención, otorgando un valor añadido a la información, que proporciona una llamada de atención a los lectores.

- Los centros hospitalarios se posicionan como una fuente de noticias para los periódicos. Este hecho queda reflejado porque gran parte de las noticias publicadas son firmadas por el propio periódico, dejando patente la importancia que tiene la institución sanitaria para los periodistas como fuente de información; esta situación nos transmite por tanto, la escasa influencia del complejo hospitalario sobre la información que se publica del mismo y la objetividad del medio.

- La noticia es el formato predominante en las informaciones publicadas, esta situación nos transmite las posibilidades y facilidades de este formato para recoger cualquier información sobre la institución, sobretodo la relacionada con los avances científicos e innovaciones en las que la institución es protagonista. El siguiente formato es el de opinión, aunque con bastante distancia, pero merecedor de una referencia, puesto que el periódico se hace eco de las opiniones de los ciudadanos y de los propios miembros de la redacción.

- Los temas principales vinculados con la información sanitaria son la innovación, los asuntos de negligencia médica y los sucesos conflictivos.

Por un lado, gran parte de los registros analizados versan sobre innovación con un fin divulgativo, constituyendo esta situación un hecho positivo para la imagen del hospital. Se le asocia con avances, adelantos, investigaciones,... otorgándoles un alcance, en la mayoría de las noticias, internacional, favoreciendo positivamente su imagen y reputación entre sus públicos.

Por otro, las situaciones de conflicto y de negligencia médica en los conjuntos totales de la temática y de la calidad informativa están íntimamente ligada al escaso control que tiene el centro sobre la canalización de la información y de no activar otras noticias, consolidándose como fuente de información

- El ámbito local-provincial es el marco en el que se circunscribe la proyección del Hospital. De esta forma, el periódico acerca de forma directa el Hospital a su radio de actuación más cotidiano, los ciudadanos a los que asiste, y cumple con una doble misión, dar respuesta a las demandas de la sociedad sobre los acontecimientos que ocurren en su medio más cercano.

- La organización, los empleados y los pacientes son los principales protagonistas de las noticias, apareciendo dichos públicos vinculados en la mayoría de las noticias.

Sociedad Latina de Comunicación Social Facultad de Ciencias de la Información - Universidad de La Laguna

Avenida César Marique, s/n; Campus de Guajara 38071 La Laguna, Tenerife (Islas Canarias - España) 
Vemos como la organización aparece ligada a sus públicos, creando un vínculo positivo. Esta estrecha relación precisa de una adecuada gestión relacional para que la tónica general sea más positiva en su reflejo mediático, pues como se ha señalado, el porcentaje de percepción negativa general de las informaciones, sobre alguno de los hospitales analizados, es bastante elevado.

- La valoración y percepción general es positiva. Podemos decir que tanto el medio como los centros hospitalarios cuentan con una relación cordial. El medio recurre a la institución sanitaria como una fuente de noticias, un lugar donde contrastar sus informaciones, y el hospital cuenta con un medio que le posibilita hacer llegar parte de sus informaciones a sus públicos. Por otra parte, podemos destacar el amplio carácter divulgativo y positivo de las noticias, vinculadas a temas de innovación y logros de la organización. Además, el medio destina grandes espacios (páginas completa) y apoya gráficamente muchas de las exposiciones. Si tenemos en cuenta estas características señaladas, sumado a que la media de notoriedad bastante alta, la imagen mediática de la organización es positiva.

- Es de destacar el caso del Hospital Puerta del Mar, sobre el que existe una percepción positiva del Hospital, pero es muy "liviana" frente al alto índice de valoraciones negativas. El margen de diferencia de la valoración positiva de las informaciones publicadas no se aleja en demasía de la negativa, ocupando esta tónica una parte sustancial del escenario mediático proporcionado por el periódico.

\section{Discusión}

Las conclusiones señaladas nos llevan a plantear una serie de posibles vías o caminos de actuación para dar un mayor impulso a la comunicación que rodea a las organizaciones y a sus públicos.

Desde los centros hospitalarios sería aconsejable afrontar ciertas medidas encaminadas a promover y gestionar la comunicación desde el propio centro sanitario, a través de su gabinete de comunicación y si éste no existiera, del responsable de comunicación, para que sea éste (el centro hospitalario) y no otra fuente, la gestora de las informaciones. De esta forma, podrían realizar una función canalizadora y despertar el interés por otros asuntos que el complejo sanitario estime oportuno dar a conocer o que pudieran ser de interés de para la ciudadanía. Además, estimamos que las organizaciones sanitarias disponen de suficientes herramientas para ello, pues se consolidan como referentes en Unidad del Dolor Agudo (UDA), técnicas reproductivas o trasplantes entre otras especialidades. Está en el propio

Sociedad Latina de Comunicación Social

Facultad de Ciencias de la Información - Universidad de La Laguna

Avenida César Marique, s/n; Campus de Guajara

38071 La Laguna, Tenerife (Islas Canarias - España) 
hospital abrir la posibilidad de ofrecer más temas que sean de interés social y de actualidad, repercutiendo en su proyección mediática.

Asimismo, se debería establecer canales para el fortalecimiento del flujo comunicativo entre la organización, es decir, el centro sanitario en su conjunto, y los pacientes/usuarios para establecer relaciones mutuas de confianza y así el centro podría enfrentar de forma más óptima las situaciones de conflicto que pudieran acontecer. Es igualmente necesario, establecer vías de diálogo con los públicos internos, ya que éstos influyen igualmente en la conformación de la imagen de la institución sanitaria, que repercutirá inevitablemente en la percepción de la sociedad.

Siguiendo las palabras de Costa Sánchez, "la prensa se interesa por la Sanidad con un espíritu de denuncia, quizá siendo consciente de que la cobertura sanitaria es una necesidad necesaria para el conjunto de la ciudadanía. Por ello, dan voz a las denuncias sociales de falta de equipamiento en hospitales, de falta de recursos humanos, de los conflictos laborales etc. Por supuesto, la explicación de este factor también ha de buscarse en el atractivo del valor noticia conflicto y la dinámica de las bad news" (2008, p.20).

Es labor por tanto de la propia institución, la gestión de una correcta y estratégica comunicación para que el interés de la prensa se despierte igualmente, hacia otros campos de actuación. Somos conscientes del escaso interés de los medios por las informaciones de carácter divulgativo, "probablemente a consecuencia de la dinámica de producción de la información que suma política y agenda del día" (Costa Sánchez, 2008, p.20); pero igualmente, "se puede dirigir la atención hacia los actos que dan origen a la noticia, para hacer uso de una función 'formativa', aportando conocimientos sobre enfermedades, sobre el sistema sanitario, líneas de investigación, etc". (Costa Sánchez, 2008, p.20). Aunque gracias a la presente investigación, descubrimos que los medios sí se interesan por las noticias de divulgación siempre que su carácter novedoso así lo merezca; por tanto, volviendo a planteamientos anteriores, serán las propias instituciones, a través de los profesionales de la comunicación, las encargadas de despertar el interés por estas informaciones, influyendo así, en la construcción de su imagen en la sociedad.

Por otra parte, también sería recomendable fortalecer y cumplir con los componentes más valorados en la comunicación médico-paciente como son el hecho de saber escuchar al enfermo, desarrollar habilidades comunicativas y de enseñanza de pautas de tratamiento a los pacientes y tener en cuenta el papel del propio paciente para participar en la toma de decisiones sobre su salud, como sostiene el Libro Blanco de la Comunicación Médica (20052010).

Sociedad Latina de Comunicación Social Facultad de Ciencias de la Información - Universidad de La Laguna

Avenida César Marique, s/n; Campus de Guajara 38071 La Laguna, Tenerife (Islas Canarias - España) 


\section{Bibliografía}

- Alcoceba Henando, J. A. (2010): "Análisis de las notas de prensa institucionales y su visibilidad en la prensa". Revista Latina de Comunicación Social 65. La Laguna (Tenerife) pp. 354-367. Recuperado el 8 de Noviembre de 2010 de http://www.revistalatinacs.org/10/art2/905 Complutense/27 Alcoceba.html.

- Almansa, A. (2010): "Relaciones Públicas y medios de comunicación. Estudio del caso español". Razón y Palabra, 70. México. Recuperado el 13 de octubre de 2010 de www.razonypalabra.org.mx.

- Arras Vota, A.M. et ad. (2008): "Comunicación y cambio organizacional". Revista Latina de Comunicación Social 63. La laguna (Tenerife) pp.418-434. Recuperado el 14 de octubre de 2010 http://www.ull.es/publicaciones/latina/08/35 79251 Chihuahua/Ana Maria Arras.html.

- Bardin, L. (1996): Análisis de contenido. Madrid: Ediciones Akal.

- Becerra Muñoz, E. (2006): Gestión de la comunicación en las instituciones sanitarias andaluzas. Málaga: Universidad de Málaga.

- Benavides, J. (2001): Dirección de comunicación empresarial e institucional. Madrid: Gestión 2000.

- Berganza, M. R. y Ruiz, J. A. (2005): Investigar en Comunicación. Guía práctica de métodos y técnicas de investigación social en Comunicación. Madrid: McGRAW-HILL.

- Bernard Krief (2005): Libro Blanco. El futuro de la comunicación médica en España y sus Comunidades Autónomas (2005-2010). Madrid: SANED.

- Camacho Markina, I. (2001): "Comunicación institucional. Funciones de los gabinetes de comunicación en la administración local". Revista Latina de Comunicación Social 44. La Laguna (Tenerife). Recuperado el 22 de octubre de 2010 de http://www.ull.es/publicaciones/latina/2001/latina44septiembre/4403camacho.htm.

- Capriotti, P. (2007). "La Responsabilidad Social Corporativa de las empresas españolas en los Medios de Comunicación". Zer 23, País Vasco, pp.61-74. Recuperado el 27 de octubre de 2010 de http://www.ehu.es/zer/zer23/ZER23 capriotti.pdf.

- Capriotti, P. (2009). "De la imagen a la reputación. Análisis de similitudes y diferencias". Razón y Palabra 70, México. Recuperado el 10 de octubre de 2010 de http://www.razonypalabra.org.mx/Articulo\%2013\%20CAPRIOTTI\%20Imagen\%20y\%20Re putacionR\&P.pdf

- Consejería de Salud de la Junta de Andalucía: http://www.juntadeandalucia.es/salud/sites/csalud/portal/index.jsp.

- Coslada Díaz, C. (2002): "Los medios de comunicación como generaciones de imagen corporativa: los públicos internos". Comunicación y Sociedad, Vol. XV, No II, Navarra, pp.

Sociedad Latina de Comunicación Social Facultad de Ciencias de la Información - Universidad de La Laguna Avenida César Marique, s/n; Campus de Guajara 38071 La Laguna, Tenerife (Islas Canarias - España) 
95-113. Recuperado el 20 de octubre de 2010 de http://www.unav.es/fcom/comunicacionysociedad/es/articulo.php?art id=345\#C01.

- Costa Sánchez, C. (2008): "Medicina y salud en la prensa. Las noticias de salud en los principales diarios de Galicia". Revista Latina de Comunicación Social 63, pp. 15-21. La Laguna (Tenerife). Recuperado el 20 de noviembre de 2010 de http://www.revistalatinacs.org/ 2008/03/Costa Sanchez.html.

- Gaines-Ross, L., (1997): "Leveraging corporate equity". Corporate Reputation Review, Vol. 1. No 1-2, Summer/Fall, p.54.

- González Borjas, A. (2004): "Salud, información periodística especializada en alza" en Ámbitos. № 11-12, Sevilla, pp. 301-310.

- Krippendorff, K. (1990): Metodología de análisis de contenido. Teoría y Práctica. Barcelona: Ediciones Paidós Ibérica.

- Losada Díaz, J. (2005): "Acercamiento al estudio de la Imagen Publicada: definición y casos", en Castillo Esparcia, A. Comunicación Organizacional, Málaga: Clave Aynadamar.

- Sánchez, F. J. (1990): "Títulos y titulares. Sobre las funciones de la titulación periodística", Comunicación y Sociedad, No 1 y 2, Volumen III, Navarra, pp. 173-183.

- Vázquez Medel, M. A. (1999). "Los medios de comunicación y la construcción social de la realidad" en Chaparro, M. (Ed.): La democratización de los medios. II Congreso de radios y televisiones locales, públicas y alternativas, Sevilla: Diputación de Sevilla, pp.207-226.

\section{Forma de citar este artículo en bibliografías}

RUIZ MORA, I y OLMEDO SALAR, S. (2011): "Imagen y medios de comunicación. El caso de los centros hospitalarios en la prensa local", en Revista PANGEA, 2, páginas 69 a 91 . Red Académica Iberoamericana de Comunicación. Recuperado el de de 2 de: http://revistapangea.org

\footnotetext{
'http://money.cnn.com/magazines/fortune/rankings/
} 